\title{
Nationwide Corporate Multimedia Network Designfor IP Based Multimedia
}

\author{
A.StephenAntoJegan ${ }^{1}$, Dr. K.P.Kaliyamurthie ${ }^{2}$, Dr.M.Sriram ${ }^{3}$ \\ \{stephen_jegan@yahoo.co.in ${ }^{1}$, kpkaliyamurthie@gmail.com², msr1sriram@gmail.com ${ }^{3}$ \}
}

Research Scholar, Department of CSE, Bharath Institute of Higher Education and Research, Chennai ${ }^{1}$, Professor, Department of CSE, Bharath Institute of Higher Education and Research, Chennai ${ }^{2}$, Associate Professor, Department of CSE, Bharath Institute of Higher Education and Research, Chennai ${ }^{3}$

\begin{abstract}
The Web Protocol (IP) Based Multimedia Network is the transmission of voice, video, information, video conferencing, web projecting and so on across IP Network. Plan of IP Based Multimedia Network is the finished manual for a triumph network plan technique. Assemble and plan a start to finish interactive media network with assistance of complete guide.This paper will cover all current key cycles, innovations and administrations and furthermore serves to

- Learn about current voice and information network foundations and transmission technologies.Understand innovation

- Requirements from both a client's and originator's viewpoint.

- Learn attempted and-tried plan tips for every innovation and service.Tune, get and distantly deal with your organization.

Contextual analysis of enormous complex corporate wide interactive media network is likewise portrayed.

- Understand fundamental and progressed convention layering ideas.

- Learn about fiber and copper link innovations. Gain a strong foundation on Multiplexing, exchanging and directing innovations and techniques. Find out about network data transfer capacity necessity and traffic designing..
\end{abstract}

Keywords: IP Network, Web Protocol, Multimedia Network, transmission of voice, video, information.

\section{Introduction}

In the professional workplace, data transfer capacity hungry and eager for processor customer worker applications have arrived at most corporate work areas. Undertaking asset arranging (ERP) apparatuses use to recover and profitability, voice-information video (interactive media) combination at the work area, expanded document sizes for data sets and clinical pictures records, colossal crosscountry and global record move and desktop video conferencing are only a couple business application heavy the requirement for additional data transfer capacity. In another time of appropriating registering, we consider that to be information move and capacity data transmission prerequisites for text, video, voice and imaging traffic increment dramatically, the organization conveyance necessities needed to move that traffic increment respectively, and no respectively[4]. 

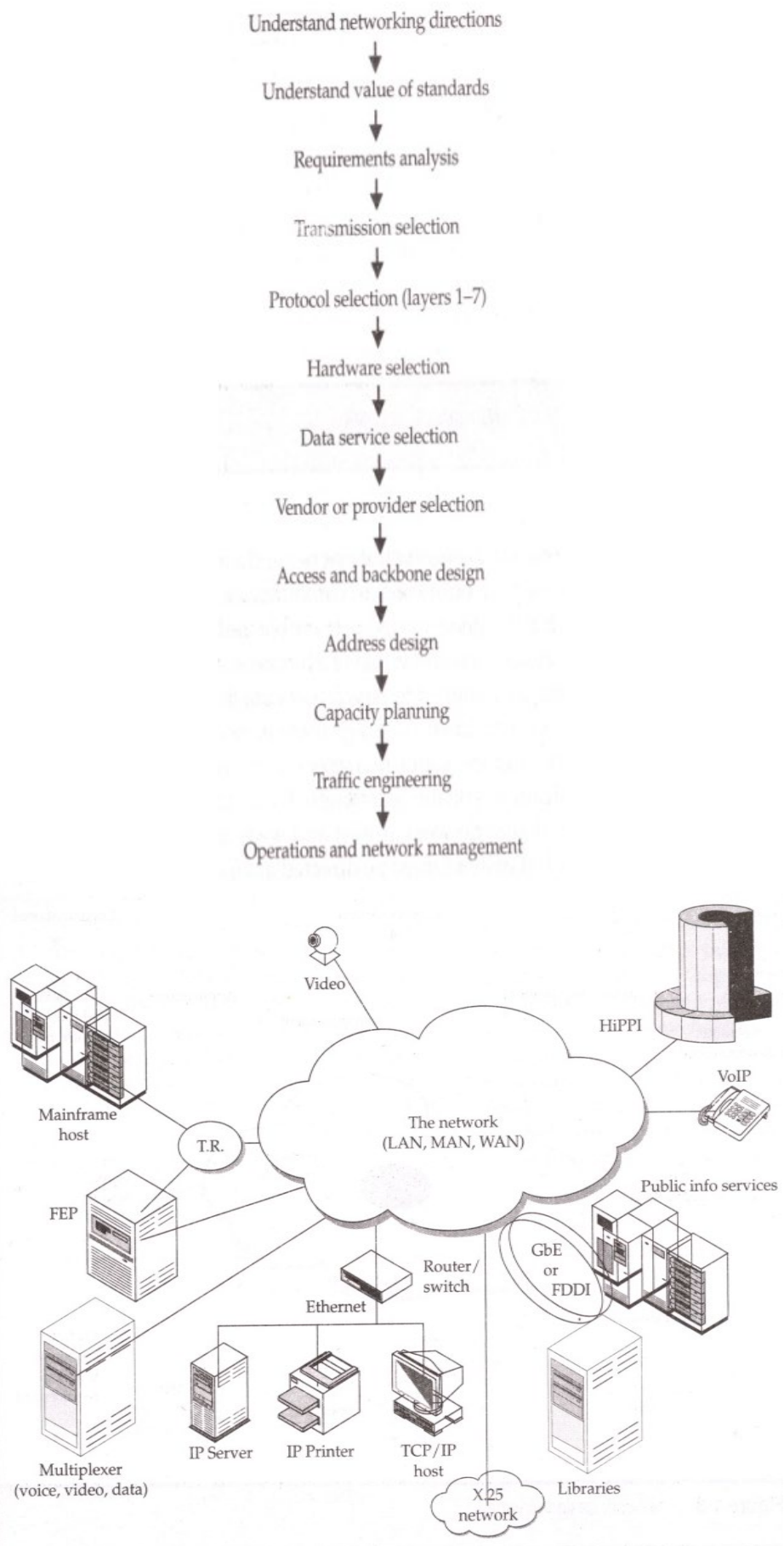

Fig. 1: IP Based Multimedia Network with user-to-network interfaces 
IP Based Multimedia Network with user-to-network interfaces is shown in figure 1 below.
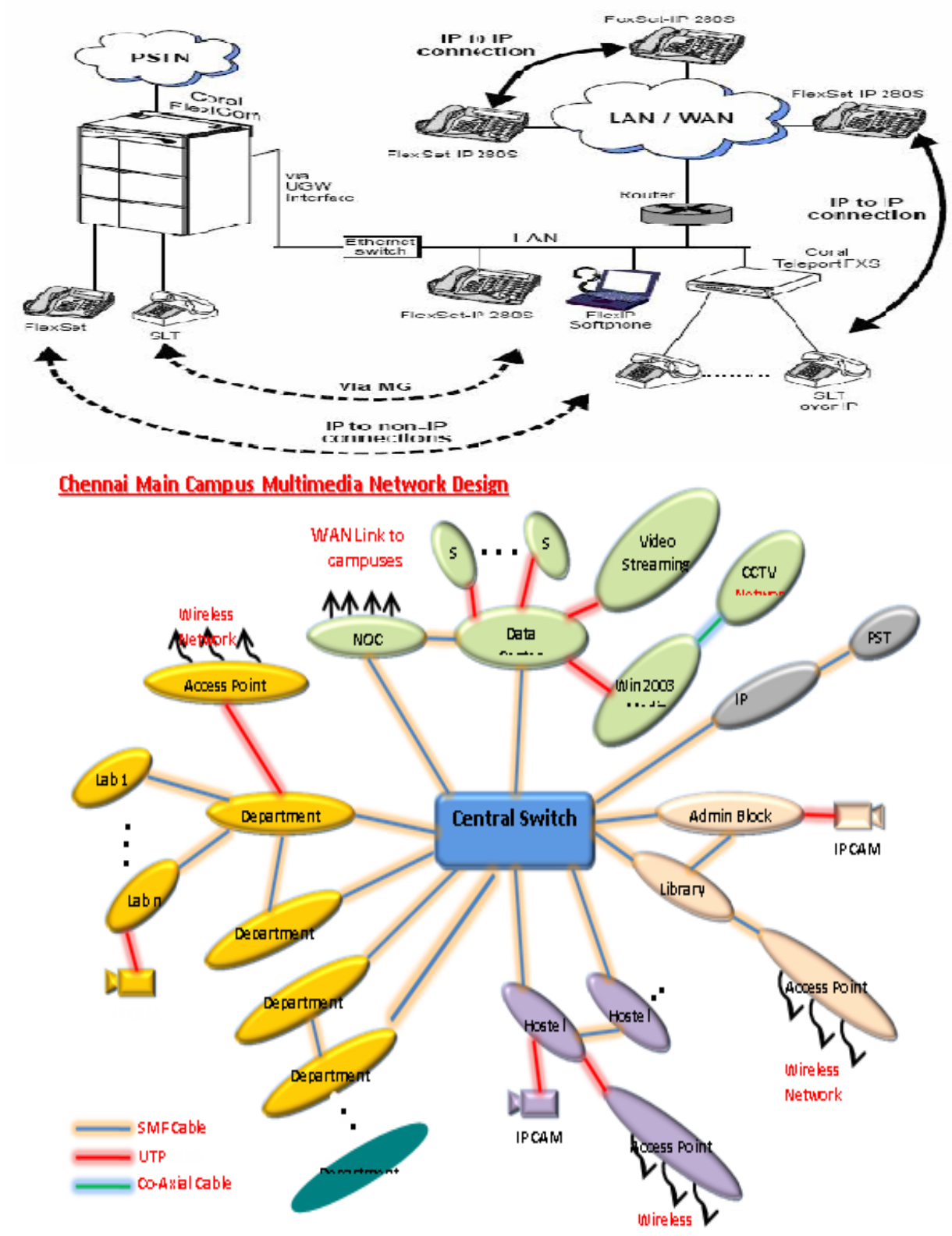

Fig. 2 : IP Based Multimedia Network design steps

The various steps of IP Based Multimedia Network design is shown in figure 2 above[5]. 


\section{Problem definition:}

Any commercial organization has least three organizations now-a-days.

- Telephone network for voice transmission.

- CCTV network for video transmission.

Information network for information transmission.

Each customer ought to have three gadgets for getting to voice, video and information. Phone for voice, TV for video and Computer for information.

A significant issue of Absence of Synchronization among the application manifests. It is additionally costly to keep up and oversee varied segments and organizations.

\section{Solution:}

Gadgets are accessible to change video and voice over to arrange. A camcorder yield can be changed over to Digital video and further to arrange parcels. Additionally gadgets, for example, IP telephones empower us to carry out Internet Telephony. In this way,

- Design of an appropriate organization engineering to incorporate the gadgets with the current IP organization would give ideal arrangement and compulsoryharmonization for the commercial application. would give the arrangement required.

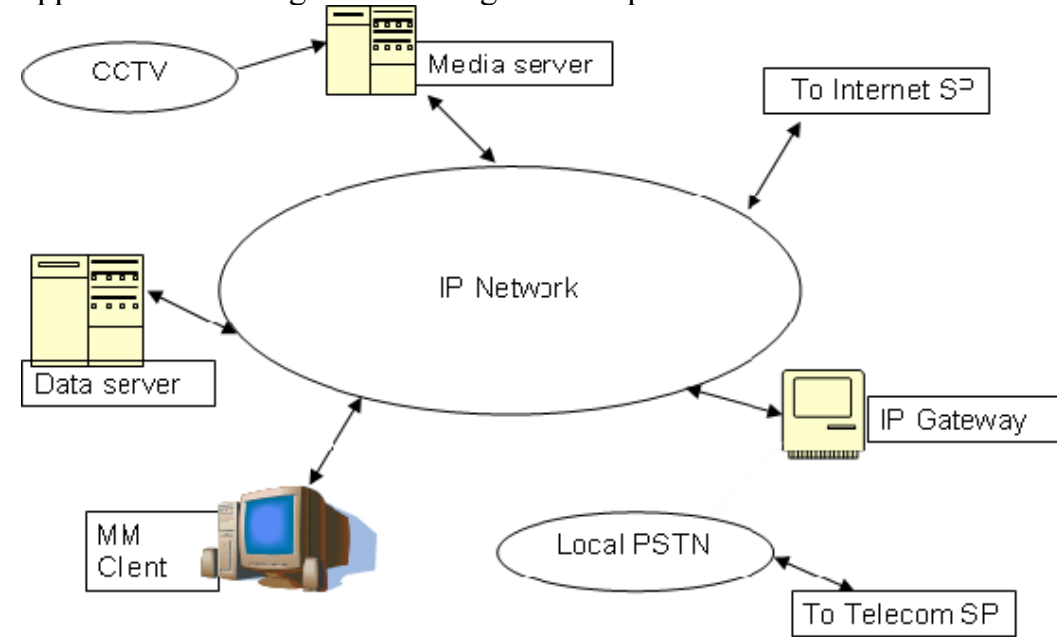

Fig. 3 Sample Multimedia network 


\section{Design considerations}

There are bunches of difficulties for information proprietor to share their information on servers or cloud. There are various answers for take care of these issues. These strategies are particularly basic to deal with key shared by the information proprietor. This paper will acquaint the confided in power with verify client the individuals who have the entrance to the information on cloud. SHA calculation is utilized by the believed position to produce the key and that key will get offer to client just as the proprietor. The believed power module gets encoded record utilizing AES Algorithm from the information proprietor and processes hash esteem utilizing MD-5 calculation. It stores key in its database which will be utilized during the dynamic activities and to decide the conning party in the framework. Believed authority send document to CSP module to store on cloud. The subsequent key sets are appeared to have various alluring properties that guarantee the classification of correspondence meetings against conspiracy assaults by other system hubs.

\section{Protocol selection:}

One potential arrangement is to move character groupings to open distributed computing stages and to demand that Cloud Service Providers process succession correlations. At present, essential grouping examination calculations are conveyed as a widespread redistributing administration on open mists. And yet, its security and protection issues are progressively rising. The re-appropriated information put away as plaintext could without much of a stretch be presented to noxious outside interlopers and inner assailants in the CSP, and the individual private data conveyed by character arrangements (e.g., individual identification, financial exchange records, hereditary markers for certain maladies, data that is utilized to recognize paternity or maternity, and so forth.) could pretty much be unveiled or mishandled. Consequently, secure re-appropriating is intended to secure the protection of character successions, and to guarantee that the booked registering demands are regularly performed on the cloud servers.

\section{Address design :}

A salted hash calculation is improved to hash the character successions and the files of cost networks, in order to protect against measurable assaults. An added substance request protecting encryption calculation is intended to encode the components of cost frameworks. Additionally, this calculation can accomplish a lack of definition under added substance requested picked plaintext assault with straight time multifaceted nature. 3) A single cloud server works for the first time to provide a security safeguarding calculable redistributing administration to viably oppose plot assaults from the cloud. With pre-processing modules of padding, partition, and extension, there is no compelling reason to decode any redistributed information in the non-intuitive grouping correlation organize. 


\section{Network architecture :}

With the quick improvement of distributed computing, the systems for safely reappropriating restrictively costly calculations are getting across the board considerations in established researchers. In the re-appropriating calculation worldview, the customers with asset compelled capacities can redistribute the substantial calculation outstanding tasks at hand into the cloud server and appreciate boundless registering assets in a compensation for every utilization way. One of the most basic functionalities in re-appropriating calculation is the certainty of the outcome. That is, the customer ought to productively check the legitimacy of the outcome returned by the cloud servers. Right now, take care of the issue of undeniable reappropriating calculation of succession examinations by coordinating the procedure of Yao's distorted circuit with homomorphic encryption. Contrasted and the current plans, our proposed arrangement empowers customers to effectively recognize the bad conduct of untrustworthy servers. Moreover, our development re-jumbles the circuit just for contorted reactions and consequently is exceptionally productive for genuine applications. Plus, we likewise present the conventional examination for our proposed development.

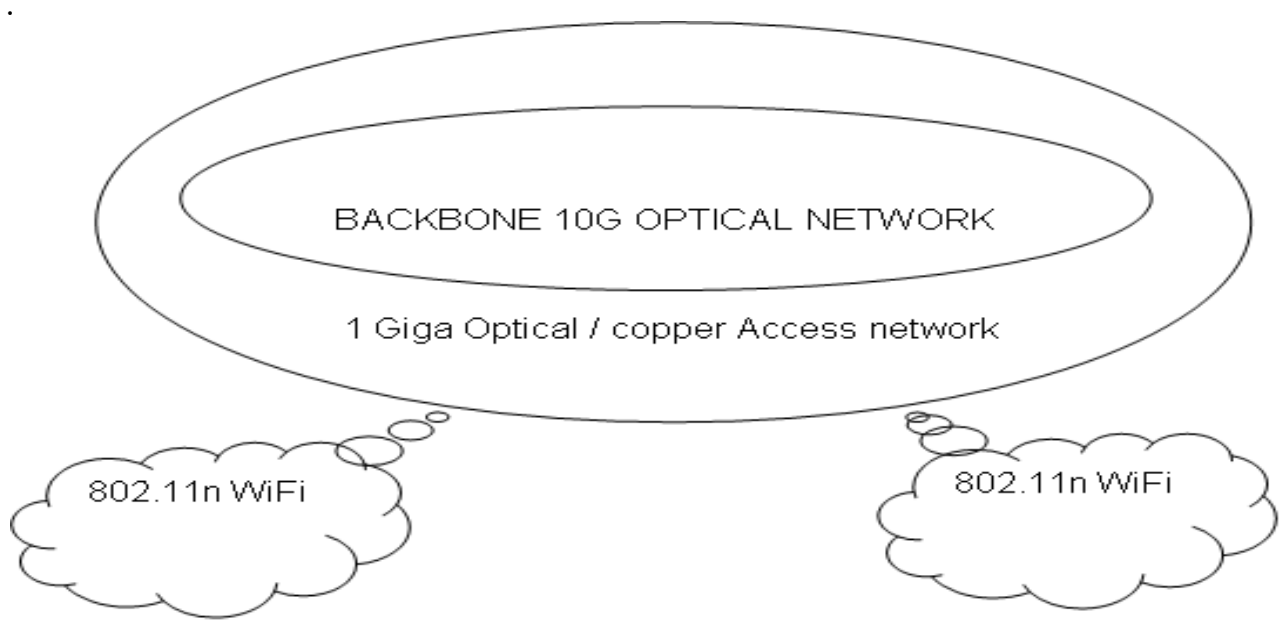

Fig. 4 Network Architecture

\section{Summary}

Through the above outline, because of the issues about the arrangement assaults that are across the board in the protected redistributing of grouping correlation calculations, this paper will acquaint the confided in power with validate client the individuals who have the entrance to the information on cloud. SHA calculation is utilized by the believed power to create the key and that key will get offer to client just as the proprietor. The believed position module gets scrambled record utilizing AES Algorithm from the information proprietor and figures hash esteem utilizing MD-5 calculation. It stores key in its database which will be utilized during the dynamic tasks and to decide the deceiving party in the framework. Believed authority send record to CSP module to store on cloud. The subsequent key sets are appeared 
to have various alluring properties that guarantee the secrecy of correspondence meetings against arrangement assaults by other system hubs.

\section{References}

[1] Multimedia networking communication protocols- Thomas C.Schmidt, tschmidt@ieee.org

[2] Data network design Darren. L. Spohn, TMH publications

[3] Delivering Voice Over IP networks Daniel Minoli\& Emma Minoli, Wiley Computer publishing.

[4] B. Balzer. Assuring the safety of opening email attachments.

[5] HouseCall. Free online virus scan. Online Publication,2002. http://housecall.antivirus.com.

[6] D. M. C. Jeffrey O. Kephart and S. R. White.Computers and epidemiology. IEEE Spectrum. http://www.research.ibm.com/antivirns/SciPapers/Kephar

[7] Dr. Kaliyamurthie K.P "An Application Of Non-Uniform Cellular Automata For Efficient Cryptography” ,Indian Journal of Science and Technology, Vol 6, Issue 5S, page 4648-4652 May 2013.

[8] Dr. Kaliyamurthie K.P "K-Anonymity Based Privacy Preserving For Data Collection In Wireless Sensor Networks", Indian Journal of Science and Technology, Vol 6, Issue 5S, page 4604-4614 May 2013.

[9] Dr. Kaliyamurthie K.P "Highly Secured Online Voting System Over Network”, Indian Journal of Science and Technology, Vol 6, Issue 6S page 4831-4836 May 2013.

[10] Dr. Kumaravel. A "vehnode: Wireless Sensor Network Platform For Automobile Pollution Control” IEEE explore, Vol Page(s): 963 - 966, 2013.

[11] Dr. Kumaravel. A "Multi- Classification Approach For Detecting Network" IEEE explore, Page(s): 1114 -1117,April 2013.

[12] T. Vijayan , M. Sangeetha , A. Kumaravel \& B. Karthik (2020): FeatureSelection for Simple Color Histogram Filter based on Retinal Fundus Images for DiabeticRetinopathy Recognition, IETE Journal of Research, DOI: 10.1080/03772063.2020.1844082.

[13] D. S. Vijayan, A. Leema Rose, S. Arvindan, J. Revathy, C. Amuthadevi, “Automation systems in smart buildings: a review", Journal of Ambient Intelligence and Humanized Computing https://doi.org/10.1007/s12652-020-02666-9

[14] Vijayan T, Sangeetha M, A. Kumaravel, Karthik B, "Gabor filter and machine learning based diabetic retinopathy analysis and detection", Microprocessors and Microsystems,2020. https://doi.org/10.1016/j.micpro.2020.103353.

[15] Vijayan T, SangeethaM, Karthik B, "Trainable WEKA Segmentation of Retinal Fundus Images for Global Eye Disease Diagnosis Application,” International Journal of Emerging Trends in Engineering Research,Vol 8, No.9, pp. 5750-5754, Sep 2020. https://doi.org/10.30534/ijeter/2020/136892020

[16] C. Amuthadevi, D. S. Vijayan, Varatharajan Ramachandran, "Development of air quality monitoring (AQM) models using different machine learning approaches", Journal of Ambient Intelligence and Humanized Computing, https://doi.org/10.1007/s12652-020-02724-2

[17] Vijayan T, Sangeetha M, A. Kumaravel, Karthik B, "Fine Tuned VGG19 Convolutional Neural Network Architecture for Diabetic Retinopathy Diagnosis," Indian Journal of Computer Science and Engineering (IJCSE), Vol. 11, No. 5, pp. 615-622 Sep-Oct 2020. DOI: 10.21817/indjcse/2020/v11i5/201105266.

[18] Vijayan T, Sangeetha M, Karthik B, "Efficient Analysis of Diabetic Retinopathy on Retinal Fundus Images using Deep Learning Techniques with Inception V3 Architecture,” Journal of Green Engineering, Vol 10, Issue 10, pp. 9615-9625. Oct 2020 